\title{
Microstructural Behavior of Nonequilibrium Systems
}

DOE/ER/ $45201--6$

James C. M. Li

Department of Mechanical Engineering

DE92 003383

University of Rochester

Rochester, New York 14627

\author{
Progress Report \\ March 1, 1989 - February 28, 1992 \\ DE-FG02-85ER45201
}

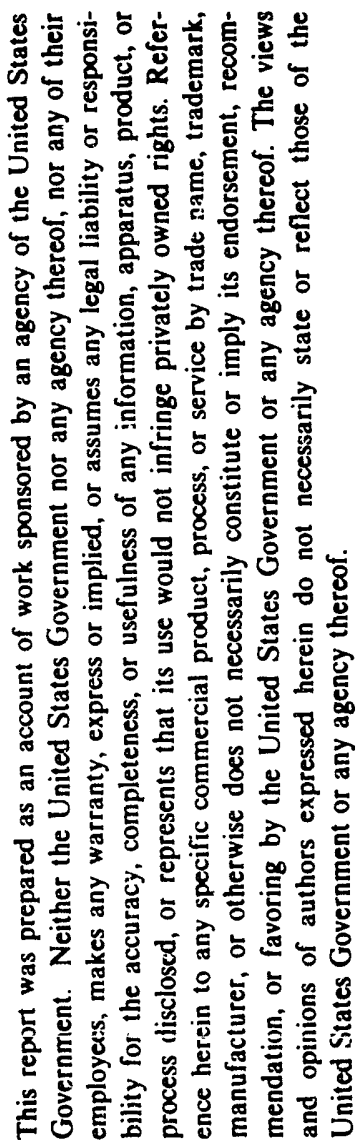

\author{
Submitted to \\ Division of Materials Sciences \\ Office of Basic Energy Sciences \\ The United States Department of Energy \\ Washington, DC 20545 \\ Attention: Dr. Joseph B. Darby, Jr. \\ and Dr. Yok Chen
}

September 1991

1 


\section{Contents}

Summary of Achievements, 1989-1992

1. Magnetic domain structure at shear bands and annealing embrittlement of metallic glass

2. Etch pits along shear bands in metallic glasses

3. Nanoporous Materials

4. Increase of Curie temperature by rapid heating

5. Dislocation-crack interactions

Nanoporous Glass $\quad 6$

$\begin{array}{ll}\text { Dislocations in Amorphous Metals } & 7\end{array}$

Fracture of Composite Materials $\quad 8$

$\begin{array}{lr}\text { Superparamagnetism } & 9\end{array}$

$\begin{array}{ll}\text { Atomic Force Microscope } & 10\end{array}$

Publications related to the Project

1. Published papers 12

2. Accepted papers 13

3. Papers submitted for publication 13

4. Abstracts for oral presentation 13

Appendices

1. Magnetic Domains Induced by Shear Bands in Metallic Glasses, Acta Metall. Mater. 38, 625-629 (1990)

2. Magnetic Domains around Annealed Shear Bands and Embrittle ment of FeBSi Metallic Glass, J. Mat. Res. 6, 371-5(1991)

3. Image Forces and Shielding Effects of a Screw dislocation near a Finite Length Crack, Mat. Sci. Eng. A142, 35-39 (1991)

4. A Finite Element Study of Stresses and Displacements near Tips of Interfacial Cracks

5. Interaction of a Screw Dislocation with an Interface Crack J. Appl. Phys. 70, 744-751 (1991)

6. Interaction of an Edge Dislocation with an Interface Crack 


\section{Summary of Achievements}

\section{Magnetic domain structure at shear bands and annealing embrittlement of metallic giass}

An important discovery in this topic area is the difference between the domain structures of shear bands formed under tension and those formed under compression. This difference was explained by the difference in the internal stresses surrounding the shear bands. In terms of dislocation models, the shear bands formed under tension may be represented by a wall of dislocations with Burgers vectors parallel to the shear step. On the other hand the shear bands formed under compression must be modelled by two sets of dislocations with Burgers vectors perpendicular to each other. This difference, not only can explain the difference in domain structutes but also can provide a reason why the material is more brittle under tension than under compression.

The kinetics of relaxation of the stress field around the shear bands as observed by the increase in domain size upon annealing revealed the difference between stress relaxation and embrittlement. Such difference provides a good explanation for the improvement of magnetic properties by rapid heating without the simultaneous occurrence of annealing embrittlement.

The foregoing is described fully in the following two papers:

1. "Magnetic Domains around Annealed Shear Bands and Embrittlemient of FeBSi Glass" J. Mat. Res. 6, 371 (1991).

2. "Magnetic Domains induced by Shear Bands in Metallic Glasses" Acta Met. et Mat. 38. 625 (1990)

The reprints are attached in the Appendix.

\section{Etch pits along shear bands in metallic glasses:}

Our effort in searching for dislocations in amorphous metals made some progress recently when Venu Lakshmanan observed some etch pits in the "edge" type of shear bands during his study of magnetic domains surrounding shear bands by the application of ferrofluids. These etch pits seem to persist after polishing them away electrolytically. It turns out that these etch pits are the result of contacting with water. 
When we changed the water-based ferrofluid into oil-based ferrofluid, no etch pits were observed. It is too bad that Venu Lakshamanan has left us to join Phillips Display Components at Ann Arbor, MI. I am looking for another student to continue this work.

\section{Nanoporous Materials:}

A major achievernent is in the nanoporous materials. I think we are the first to discover the large damping properties associated with fluid flow inside the pores. The fluid is first adsorbed on the inner surfaces of the pores and then fills up the pores. The adsorbed layer does not contribute to the damping peak which is caused by the flow of the filled up fluid. The pressure gradient created by bending is the driving force for the fluid flow. The peak frequency shifts toward lower values at low temperatures and less fluid contents, consistent with the mechanism. The effective viscosity for the flow of fluid inside the pores is about 3 times of the bulk viscosity which agrees with the result of other measurements.

\section{Increase of Curie temperature by rapid heating:}

Another discovery which may be very important also is the increase of Curie point by rapid heating. Usually the Curie pcint, the temperature at which ferromagnetism is transformed into paramagnetism, is considered constant like the melting point. It may increase somewhat with increasing heating rate but the increase is considered to be only a fraction of a degree. However, we discovered at $60^{\circ} \mathrm{C} / \mathrm{sec}$, the Curie temperature can increase by as much as $120^{\circ} \mathrm{C}$ for $\mathrm{Fe}_{78} \mathrm{~B}_{13} \mathrm{Sig}_{9}$ (Metglas 280552). This may be important in optical recording devices because there the heating rate is of the order of $10^{5} \mathrm{C} / \mathrm{sec}$. Someone at Kodak was interested and we negotiated a research support in the form of a fellowship. Unfortunately at the time I did not have a proper student so the feliowship went to the Chemical Enginearing Department. Nevertheless we will continue to look into this effect.

\section{Dislocation - crack interactions:}

We made several advances in this area. First of all the analytical solution for an interface crack under load is simply wrong despite of its widespread usage because of the overlapping displacement inside the crack. However, we checked the analytic solution by 
the finite element method and found that the solution is basically correct except for the boundary conditions. Then we modified the boundary conditions in the numerical method and found some interesting results. A preprint is attached in the Appendix.

Then we solved the problem of the interaction of both a screw dislocation and an edge dislocation with an interface crack. The case for the screw dislocation has been published already. A reprint is attached in the Appendix. The manuscript for the case of the edge dislocation is also attached in the Appendix. The important result is the differentiation between the dislocations emitted from the crack and those emitted elsewhere and then moved to the vicinity of the crack. Such differentiation has not been pointed out clearly before and the interactions in the two cases are entirely different. These results certainly have a bearing on the strength of composite materials.

The third achievement relates to the region of accelerated fatigue crack growth. While we were examining the effect of loading wave form, we discovered something new, namely, in the region of accelerated fatigue crack growth, dislocations are no longer emitted from the crack. Instead, the emitted dislocations in front of the crack moves with the crack in a steady state situation. It turns out that the steady state is stable, namely if the crack is constrained not to propagate so that the plastic zone grows somewhat and then the crack is allowed to grow, the plastic zone will shrink back to the steady state size. We were somewhat surprised because the region of accelerated crack growth is usually considered as unstable. We are writing this up.

Another finding with respect to the fatigue crack propagation is the overloading effect and the effect of obstacles in the crack plane. We discovered to our dismay that there is no overloading effect in fatigue crack propagation under Mode II or Mode III loading. After many repeated simulations with different parameters, we finally are convinced that this must be the case. Fortunately we found someone who could perform the experiment and sure enough the results agree with our findings. We are writing these up. 


\section{Nanoporous Glass}

As mentioned in the last progress report, we examined some properties of the nanoporous glass readily available from our neighbor - Corning Glass Corporation. This material has about $30 \%$ voids in a range of sizes between 4 and $15 \mathrm{~nm}$. Like a sponge, water can be absorbed into the material within a minute or so and can be removed by heating in a furnace. For the amount of water absorbed, the $30 \%$ void volume is completely filled showing that isolated voids are rare or non-existent.

When we made internal friction measurements on the as-received porous glass, nothing exciting was seen. The internal friction was small and had no frequency dependence. However the water-filled porous glass did show larger internal friction and a well-behaved peak at about $-80^{\circ} \mathrm{C}$. We first suspected that perhaps $-80^{\circ} \mathrm{C}$ is the freezing point of water inside these small cavities. But later we found out that this could be caused by a piezoelectric transition of ice. The freezing point of water in these small cavities is about $10 \mathrm{C}$ lower than the regular freezing point. At room temperature there is an internal friction peak related to the flow of water between the small cavities. This part of the study is being written up.

The water inside the porous glass seems to be separable into two types. When the water content is small, the water is adsorbed on the inner surfaces of glass pores. Such water may not flow during bending. The other type of water fills up the pores and will flow under the pressure gradient created by bending. The first type amounts to about $60 \%$ of the saturation and the second type $40 \%$. The internal friction peak disppears when the water content is less than $60 \%$ of saturation. When the water content is more than $60 \%$, the peak height increases with increasing water content until it is $100 \%$ saturaied. As a function of frequency, the peak shifted to lower frequencies for less amount of water in the pores or for lower temperature, all consistent with the flow of water as the damping mechanism. From the energy loss in damping the viscosity of water is found 3 times larger than bulk water. This should be the result of the small size of the pores. The effective viscosity of water inside larger pores will be studied also.

The $60 \%-40 \%$ separation must depend alse on the average size of the pores. Several more pieces of porous glass with larger pores will be used to see at what fraction of saturation the peak disappears. This dependence will enable us to determine the thickness of the adsorbed layer which has limited mobility. 


\section{Dislocations in Amorphous Metals}

We have had no success in the attempt to see dislocations by direct observation of the magnetic domain structure. People at IBM and NIST are still too busy to work on this. One time we even invited an IBM scientist to give a seminar. She was interested in what we proposed to do but had no time to do it. In view of the important applications of the atomic force microscopy and our continued interest in the deformation mechanism of amorphous metals and alloys, we are attempting to construct an atomic force microscope ourselves. This is not entirely impossible because we have a group in our Chemistry department actually working on this and we will benefit from their experience. They have a few designs which seem to work and we offer to make a new design based on their old models but to include several improvements relevant to our goals. While we are making our own atomic force microscopes, we encountered Dr. David Abraham of IBM who is willing to look at our samples in his magnetic force microcope. We have already sent him the samples and are waiting for some nice pictures from him.

The etch pits we observed along the "edge" type shear bands are very encouraging and it is too bad that Venu Lakshmanan has left so I have to find somebody else to work on it. As mentioned in the proposal submitted earlier, we have a couple of ways to reveal the etch pits or magnetic domains under high resolution. All these have not got a chance to be carried out yet. The behavior of the etch pits, if they are related to dislocations, is obviously very important. Fortunately we are going to have some free time in the Hitachi laboratory through the kindness of Dr. Kazuyuki Koike to use their SEM with magnetic spin polarization analysis. One of my visiting scholars will travel to Japan to perform this study. This technique should reveal in greater detail the magnetic domain structure surrounding the shear bands as well as the magnetization direction in each domain. Hopefully we will be able to see dislocations in amorphous metals. 


\section{Fracture of Composite Materials}

The problem is more complicated than we originally anticipated. The main cause is that the existing analytic solution for a finite interface crack has an oscillatory displacement field in which the material inside the crack can overlap, a non-physical result. Because of this we really could not solve the problem involving the interaction between an edge dislocation and an interface crack. Fortunately for the screw dislocation under Mode III loading this oscillating behavior is absent and so we solved that problem. The conclusions are probably applicable qualitative!y to the situation of edge dislocations under Mode I or Mode II loading. Numerically we did confirm the analytic solution and went on to solve the problem without oscillatory behavior and without material overlap. However without an analytic solution, there is too much trouble to analyze the interaction between an edge dislocation and an interface crack. What we could do is to select a couple of qualitative behavior from the results of the screw dislocation interface crack interaction and confirm them numerically. We have nol done that yet.

For a homogeneous medium we made progress in the dislocation - fatigue crack interactions. In an attempt to see the overloading effect in fatigue crack propagation, we first applied a large stress intensity factor to develope a large plastic: zone and then suddenly the applied stress intensity factor is reduced. We expected a much reduced crack propagation rate because of the large shielding effect of the plastic zone. To our surprise the crack propagation rate only gradually decreased to the new value correspoding to the smaller stress intensity factor in Mode II or Mode III loading. We repeated several times using many different parameters including the introduction of obstacles in the plane of the crack so as to prevent the disappearance of the plastic zone into the crack during unloading. The results were all the same. So we were forced to conclude that there is no overloading effect in Mode II or Mode III fatigue crack propagation. Then we had an opportunity to suggest the very experiment to be carried out at the Nanjing Institute of Chemical Technology because they had the proper equipment and they already observed some pure Mode II fatigue crack propagation. Well you guessed, the experiments did confirm our computer simulation. We are writing this up. Because of this we plan to continue this coopera'ion and I am inviting the person involved in the experimentation to visit us for a short period. 


\section{Superparamagnetism}

The apparent super paramagnetism observed in amorphous metals by superposing an ac current received some attention from people in industry. Obviously there may be some application of this phenomena. Real superparamagnetism from nanocrystalline iron has not been tried because of the lack of very fine (a few nanometers) iron powders. The powders we made ourselves and those we could purchase were not fine enough for this purpose. As a result, we have been putting this project on hold until we have some fine powders to work with. Currently we are cooperating with the people in the Pacific Metals Co., Lid., Tokyo, to provide us with some very fine iron powders which they can produce in a limited way. We have some of their powders now and we may try to make some samples for superparamagnetic measurements. We are also cooperating with the Navy Research Laboratory to let us use some of their powders made by an organic reaction. But we have not obtained their powders yet. A joint proposal between the Naval Research Lab and the University of Rochester is being prepared to be submitted to the Office of Naval Research. 


\section{Atomic Force Microscope}

As mentioned in my last year's proposal, we are constructing an atomic force microscope (AFM) ourselves with the help of cur Chemistry Department. It is partly done but there are still some details to work out. The idea is to change it into a magnetic force microscope later so we can see the magnetic domain structure around the shear bands. We are also fortunate to collaborate with Dr. David Abraham of IBM to try our experiments on his magnetic force microscope first. At least this will help us determine whether our microscope will be as good as his.

The AFM is capable of mapping forces near the surfaces and hence the distribution of forces around surface defects such as a shear band or a particle. Like the Scanning Tenneling Microscapy (STM) the AFM is capable of extremely high resolution in three dimensions. In the AFM, minute forces near the surface of the sample act on the tip of a cantilever stylus system. These forces cause very small deflections of the cantilever which are detected with sub-angstrom sensitivity by an optical displacement detector. By using a magnetic needle, the AFM can be used to probe minute domain structures around shear bands. We have high hopes that this technique will allow us to see dislocations in amorphous metals.

The basic operating method of the AFM is simple. A Piezoelectric (PZT) scanner with the sample mounted on it rasters under the tip which is kept motionless. Fine pitched screws are used for coarse height control to position the sample near enough to the tip that the interacting forces cause a deflection in the cantilever. This is detected by the optical detection system which then sends a signal to the electrical feedback loop that drives the PZT in the $Z$ direction. Raster scanning of the surface is then done at a fixed deflection of the cantilever or in other words at constant force between the tip and surface. The image is reconstructed on a computer from the PZT heights.

In order to detect very small forces $\left(10^{-6} \mathrm{~N}\right.$ to $\left.10^{-12} \mathrm{~N}\right)$, a cantilever has to be very soft, ideally with a spring constant between $10^{-2} \mathrm{~N} / \mathrm{m}$ to $10^{2} \mathrm{~N} / \mathrm{m}$. In addition, in order to achieve imaging bandwidths comparable to that obiainable in STM, AFM cantilevers should have resoriant frequencies $>10 \mathrm{kHz}$. To satisfy both of the above requiremenis, the mass of the cantilever must be reduced significantly. Apart from this the cantilever should have high lateral stiffness in order to reduce the effects of lateral forces in AFM. Thus the cantilever is limited to certain geometrical constraints. Finally a sharp protruding tip must be formed at the end of the cantilever to provide a well defined interaction with the sample over a small area. The tip should be at the lowest 
point of the cantilever system with sufficient height to provide good clearance for approaching rough surfaces. For repulsive mode imaging, the microscopic structure of the point of the tip is important for high resolution microscopy since the contact area between the tip and sample involves only a few atoms. On the other hand, in attractive mode imaging a larger volume of the tip is involved, thus the macroscopic shape of the tip is also important.

The AFM uses the Optical Detection Method (Beam Bounce Version) for determining the displacement of the cantilever. In the beam bounce configuration, small displacements at the end of the cantilever are translated to much larger displacements of the diode laser beam due to the large path length between the point of reflection off the cantilever and the Position Sensitive Detector (PSD). With a 100 misron long cantilever, a path length of $4 \mathrm{~cm}$ will give a magnification of 400 times. Thus a one angstrom displacement of the cantilever translates to a 400 angstrom position change on the cantilever. Since the sensitivity of the PSD's are about 50 angstroms, the beam bounce technique can theoretically provide vertical resolutions of up to 0.1 angstroms.

The AFM being built is we!l en is way to completion. The mechanical set up is nearly done and only the electronics remain to be built. Access to the complicated computer programs necessary for imaging has already been granted, but an adequate computer system has not yet been purchased. Once all of the equipment has been assembled, the next step will be to troubleshoot it. As I mentioned earlier, we have high hopes that this instrument will give us the kind of magnification we need for our purposes. 


\section{Publications Related to the Project}

I. Published papers: (not included in the last 3-year summary)

1. Recovery Processes in Amorphous Polymers, J. Mat. Sci. 22, 3620-3630 (1987)

2. Negative Creep and Mechanochemical Spinodal in Amorphous Metals, Mat. Sci. Eng. 98, 465-468 (1988)

3. Structure of Magnetic Domains surrounding Metallic Glass Shear Bands, Mat. Sci. Eng. 98, 483-386 (1988)

4. Edge Dislocations Emitted along Inclined Planes from a Mode I Crack, Mat. Sci. Eng. A104, 95-104 (1988)

5. A Theoretical Limit of Fracture Toughness, Scripta Met. 22, 837-838 (1988)

6. Dynamic Simulation of Edge Dislocation Arrays ahead of a Mode I Fatigue Crack, Science at the John von Neumann National Supercomputer Center, 1988 Annual Report, pp109-112

7. Anisotropic Methanol Transport in PMMA after Mechanical Deformation, Polymer 29, 1221-1226 (1988)

8. Summation of Infinite Series Related to Roots of Certain Functions, Int. J. Math. Ed. Sci. Tech. 19, 89-93 (1988)

9. Stored Energy of Cold Work in Polystyrene, Polym. Eng. Sci. 28, 1198-1202 (1988)

10. Analysis of Endothermic Peak in an Annealed Metallic Glass, J. Non-Crystalline Solids, 107, 225-232 (1989)

11. The Effect of Grain Size on Fracture Toughness, Phil. Mag. A59, 1245-1261 (1989)

12. An Attempt to Verify the Orowan Equation, Current Contents,29, No.39, p. 12 (1989)

13. Magnetic Domins Induced by Shear Bands in Metallic Glasses, Acta Met. et Mat. 38, 625-629 (1990)

14. The Effect of Core Traction on the Image Force of a Screw Dislocation, Mat. Sci. Eng. A123, 201-206 (1990)

15. Microstructural Control through Strategic Materials Processing, Ashby Symposium: The Modelling of Materials Behavior and Its Relation to Design (Ed. by J. D. Embury and A. W. Thompson, TMS 1990) pp 149-173

16. Dislocations Emitted from a Fatigue Crack I. Identical Burgers Vectors, Mat. Sci. Eng. A129, 167-174 (1990) 
17. Dislocations Emitted from a Fatigue Crack II. Burgers Vectors of Both Signs, Mat. Sci. Eng. A129, 175-182 (1990)

18. Magnetic Domains around Annealed Shear Bands and Embrittle ment of FeBSi Glass, J. Mat. Res. 6, 371-375 (1991)

19. Second Order Kinetics of Enthalpy Recovery in Polystyrene, J. Polym. Sci. Part A. Polym. Chem. 27, 1125-1136 (1989)

20. Interaction of a Screw Dislocation with an Interface Crack, J. Appl. Phys. 70, 744-751 (1991)

21. Image Forces and Shielding Effects of a Screw Dislocation near a Finite Length Crack, Mat. Sci. Eng. A142, 35-39 (1991)

II. Accepted Papers:

1. image Forces and Shielding Effects of an Edge Dislocation near a Finite Length Crack, Acta Met. et Mat.

III. Papers submitted for publication:

1. A Finite Element Study of Interfacial Cracks

2. The Interaction of an Edge Dislocation with an Interface Crack

3. Vacancy Clusters in FCC Crystals

4. Dislocation Emission from Grain Boundaries during rapid Heating and Cooling

5. A Nanocavity in a FCC Crystal

IV Abstracts for oral presentations

1. "Dislocation Free Zone in Front of a Fatigue Crack" TMS Annual Meeting Abstracts, 1989, p. 7. (with Jia Li and V. Lakshmanan).

2. "Effect of Heating Rate on the Curie Temperature of an Amorphous Alloy" TMS Fall Meeting Abstracts, 1989, p. 57 (with Der Ray Huang).

3. "Vacancy, Interstialcy and Nanocavity inside a FCC Crystal" ibid, p. 60 (with Y.C. Lian).

4. "Interaction of a Screw Dislocation with an Interface Crack" ibid, p. 79 (with Tong-Yi Zhang).

5. The Fractal Nature of Craze Pattern Developed in Mode I Fracture of Polymeric Materials" MRS Fall Meeting, 1989, Final Program and Abstracts, p. 637 (T 307).

6. "The Effect of Core Traction on the Image Force of a Screw Dislocation Ernitted from a Crack" Irsee Conf. on Fundamentals of Fracture, 1989, pp. 58-69 (with Tong-Yi Zhang).

7. "On Image Dislocations Inside a Finite Crack" ibid, pp. 70-71 (with Tong-Yi Zhang).

8. "Dislocation Emitted from a Propagating Fatigue Crack" ibid, pp. 105-106 (with Jia Li). 
9. "A New Approach to Fracture Toughness Testing" TMS Fall Mitg Abstracts, 1990,p. 14 (with Beta Yuhong Ni and Tong-Yi Zhang)

10. "Dislocation Emission from a Fatigue Crack" ibid, p. 38 (with Jia Li)

11. "Photoelastic and Finite Element Analysis of Solid Spheres in Loading

Contact" ibid, p. 40 (with C. W. Shih and P. D. Funkenbusch)

12. "A High Damping Consolidated Ultra Fine Powdered Material" ibid, p.53 (with Y. C. Lian)

13. "Internal Friction of Nanoporous Glass" ibid, p. 53 (with Zhongshan Chen)

14. "Damping Properties of Dealloyed Porous Copper" ibid, p. 53(with Un-Sig Min)

15. "Computer Simulation of Powder Packing" TMS Fall Mtg Abstracts, 1991, p. 6 (with Chun-Wei Shih)

16. "Tip Effect of Nanoindentation" ibid, p. 25 (with Chun-Wei Shih)

17. "Creep Cavitation in Front of Mode I Crack" ibid, p. 56 (with Beta Yuhong $\mathrm{Ni})$

\section{Appendices}

1. Magnetic Domains Induced by Shear Bands in Metallic Glasses, Acta Metall. Mater. 38, 625-629 (1990)

2. Magnetic Domains around Annealed Shear Bands and Embrittle ment of FeBSi Metallic Glass, J. Mat. Res. 6, 371-5(1991)

3. Image Forces and Shielding Effects of a Screw dislocation near a Finite Length Crack, Mat. Sci. Eng. A142, 35-39 (1991)

4. Interaction of a Screw Dislocation with an Interface Crack J. Appl. Phys. 70, 744-751 (1991)

5. A Finite Element Study of Stresses and Displacements near Tips of Interfacial Cracks

6. Interaction of an Edge Dislocation with an Interface Crack I. Stress and Displacement Fields

7. Interaction of an Edge Dislocation with an Interface Crack II. Image Force and Shielding Effects

* not riseuded 

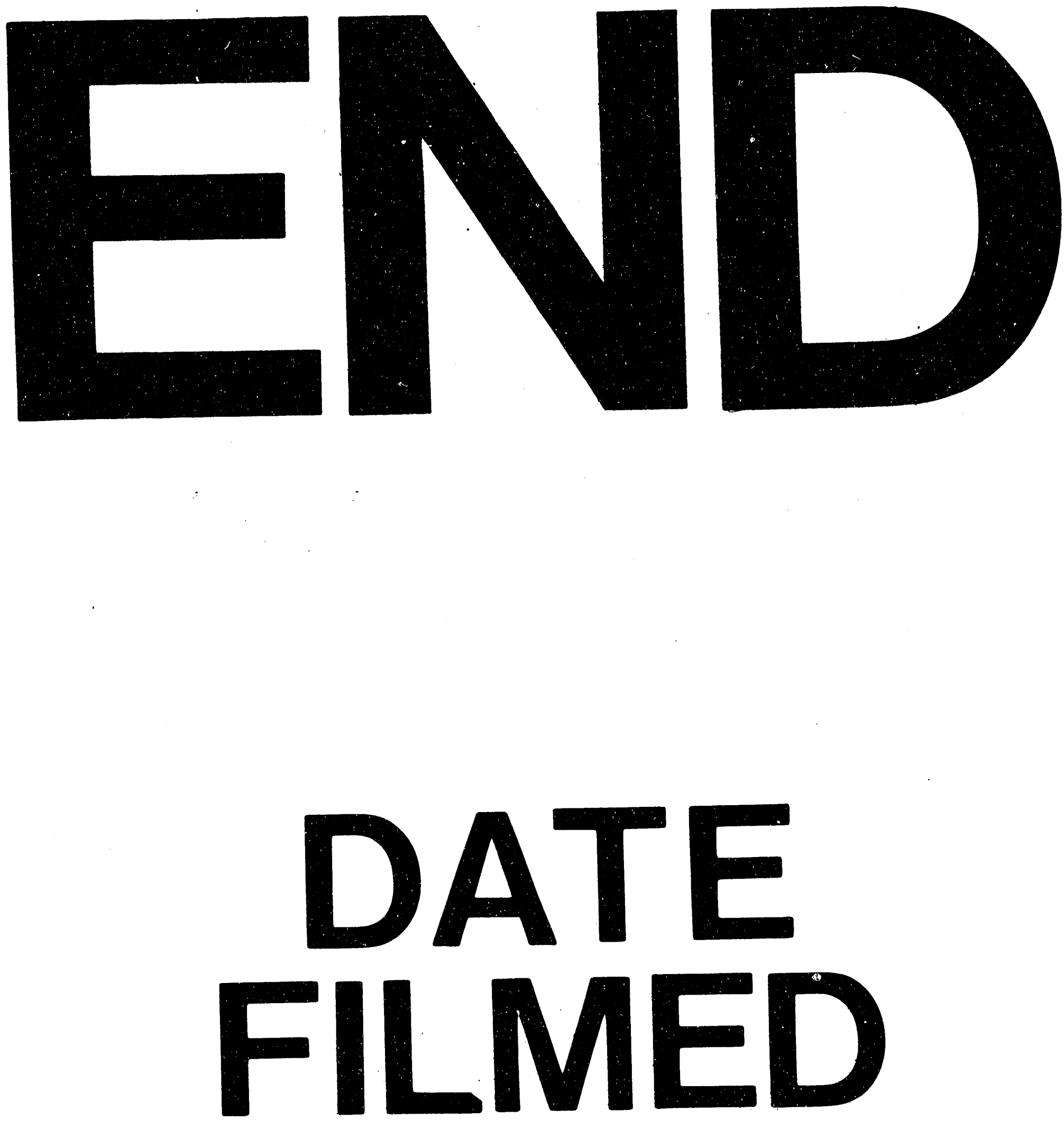

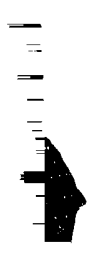

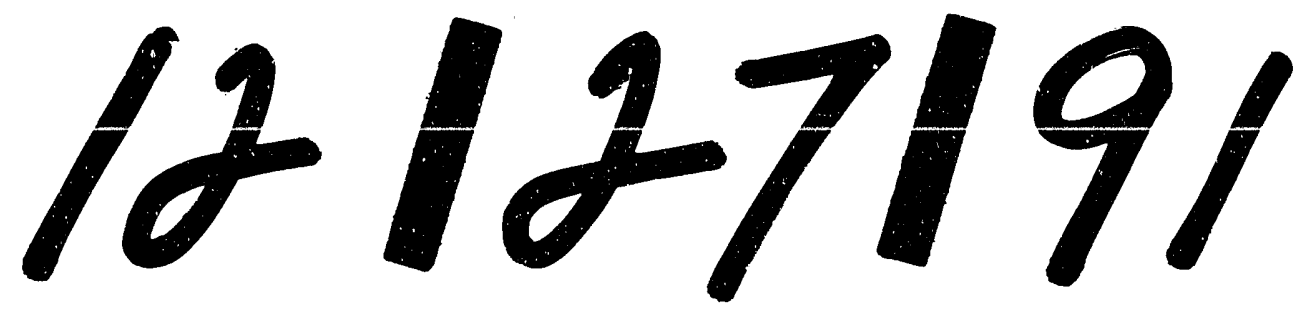

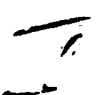


